\title{
Enabling Micro-Entertainment in Vehicles Based on Context Information
}

\author{
Florian Alt, Dagmar Kern, Fabian Schulte, Bastian Pfleging, \\ Alireza Sahami Shirazi, Albrecht Schmidt \\ Pervasive Computing and User Interface Engineering \\ University of Duisburg-Essen \\ Schützenbahn 70, 45117 Essen, Germany \\ \{florian.alt, dagmar.kern, bastian.pfleging, alireza.sahami, albrecht.schmidt\}@uni-due.de \\ fabian.schulte@stud.uni-due.de
}

\begin{abstract}
People spend a significant amount of time in their cars (US: 86 minutes/day, Europe: 43 minutes/day) while commuting, shopping, or traveling. Hence, the variety of entertainment in the car increases, and many vehicles are already equipped with displays, allowing for watching news, videos, accessing the Internet, or playing games. At the same time, the urbanization caused a massive increase of traffic volume, which led to people spending an ever-increasing amount of their time in front of red traffic lights. An observation of the prevailing forms of entertainment in the car reveals that content such as text, videos, or games are often a mere adaptation of content produced for television, public displays, PCs, or mobile phones and do not adapt to the situation in the car. In this paper we report on a web survey assessing which forms of entertainment and which types of content are considered to be useful for in-car entertainment by drivers. We then introduce an algorithm, which is capable of learning standing times in front of traffic lights based on GPS information only. This, on one hand, allows for providing content of appropriate length, on the other hand, for directing the attention of the driver back to-wards the street at the right time. Finally, we present a prototype implementation and a qualitative evaluation.
\end{abstract}

\section{Categories and Subject Descriptors \\ H.5.1 [Multimedia Information Systems]}

\section{General Terms}

Performance, Human Factors

\section{Keywords}

Micro entertainment, vehicle, GPS, context

\section{INTRODUCTION}

For many people cars are an integral part of everyday life. Statistics of the U.S. Department of Transportation ${ }^{1}$ show that Americans spend on average 86 minutes per day in their vehicles (Europe: 43 minutes), most of the time alone. This raises demands for entertainment, which, in recent years, fostered the development of living room like conditions inside vehicles.

\footnotetext{
${ }^{1}$ BTS: http://www.bts.gov/data_and_statistics/(accessed 5/2010)
}

Copyright held by author(s)

AutomotiveUI'10, November 11-12, 2010, Pittsburgh, Pennsylvania ACM 978-1-4503-0437-5
In contrast, especially in highly populated areas, the ever-growing amount of vehicles led to a significant increase of waiting time in traffic jams and in front of traffic lights. Densely occupied roads require a high degree of attention by the drivers, which makes it difficult to provide appropriate means for entertainment without distracting the driver from his primary task, that is driving [10].

For entertainment, similar to all other secondary tasks in a vehicle, road safety is crucial and hence a limiting factor. Nowadays, audio content, such as CDs or radio, is among the most popular forms of entertainment due to its rather ambient character. On the other hand, visual content requires a high level of attention and has a high potential to distract drivers. In many countries it is thus prohibited to watch video content such as television programs or movies while the vehicle is in motion and legislation requires mechanisms to strictly enforce this (e.g., turning off the screen on the hardware side once travelling faster than $3 \mathrm{~km} / \mathrm{h}$ ). Yet, more and more cars are equipped with video hardware such as monitors allowing for displaying content such as text, videos, emails, or even playing games.

Perceiving or engaging with visual content in the vehicle significantly differs from doing so at home. Yet, traditional content still prevails inside vehicles. We believe that the type of content shown on such screens has a strong influence on the perception and hence the degree of distraction from the driving task. This is due to several reasons: first, the user's attention is taken off the road and focused on the screen. Since the display is not aware of the current situation on the street, it cannot provide any indication when the attention of the driver should be directed back towards the road (e.g., when a traffic light turns green or when a railroad crossing is passable again). Second, content such as television programs, movies, and also games are in general not suitable to be (entirely) perceived in very small time frames.

In this paper we show how to enable in-car micro-entertainment based on the use of a car's context information. Our concept allows for adjusting multimedia content in a way such that (1) the length of the content can be tailored to the time frame available while waiting at a traffic light, and (2) the attention of the user can be directed back to the street once the traffic light is expected to turn green again. Our algorithm learns standing times by capturing and aggregating data from (multiple) vehicles and provides this information to any entertainment system either in real-time or via a local database. People commuting daily often take similar routes at the same time of the day (e.g., in the morning / evening). This makes it possible to derive estimates of waiting times based on large amounts of collected GPS information. However, we show that also for initial use, a good approximation can be achieved. 
The focus of this paper is on the enabling technologies and is considered to be the first step towards a micro-entertainment enabling infrastructure. Though we provide some qualitative data it is not in the scope of the paper to focus on the impact on users' driving behavior. This is work that is currently being undertaken in the lab and that shall be reported on in the future.

We make the following contributions: (1) We report on the findings from an online survey among 127 participants on the potential of micro-entertainment. (2) We present an algorithm, which identifies zones in front of traffic lights and associates an average waiting time depending on data obtained both from former encounters and other vehicles. (3) We present a prototype implementation and a qualitative evaluation of the enabling technology.

\section{BACKGROUND AND RELATED WORK}

In-car entertainment goes back into the 1930s when the first commercial automobile radios entered the market and nowadays includes different communication channels and displays hence providing a similar set of information as can be found in home entertainment. In the following we report on entertainment in cars, available sensors and their applications, and car communication.

\subsection{Entertainment / Infotainment in Cars}

In vehicles different devices are used to convey information via various communication channels. Most popular devices, such as radios and $\mathrm{CD}$ players, mainly use the audio channel hence limiting the distraction of the driver. Content includes music, news, audio books, ads, and traffic information. Mobile phones, often connected to cars' internal speaker systems via Bluetooth, do not only allow for interpersonal communication but also provide a convenient way of connecting to the Internet. This enables access to up-to-date traffic information, emergency services, and emails. Furthermore, multimodal displays also allow for showing video content via DVB-T, Video on Demand, or DVD. However, in many countries law permits the perception of video content while driving. As a result, displays are often integrated in the headrest of front seats, providing content to passengers in the back seats only.

Whereas Laurier et al. [18] reported on what people do wile travelling in general, different research projects have investigated the use of in-car entertainment systems. In [1] a context-based entertainment system was implemented, using a cab's context information to provide adaptive contents. In [19] an online game based on multi-hop wireless communication is presented, allowing for a shared user experience among travelers on the same road. Gustafsson et al. [11] presented a context-aware storytelling game aiming at creating a narrated experience for children in the backseat. CommuterNews [21] aims at engaging the user into interaction with an in-car entertainment system. Kamp et al. presented a usability assessment of an in-vehicle Internet browser [15].

Further, the impact of in-car entertainment on the user behavior has been subject to research [8]. Harbluk et al. [11] reported that even if in-vehicle devices are hands-free, significant changes in the drivers' behavior might occur due to the cognitive distraction. To control entertainment systems research focused on how to provide access and control in an easy and intuitive way. Prominent examples are multi-functional interfaces such as BMW's iDrive ${ }^{2}$,

\footnotetext{
${ }^{2}$ Faszination BMW - iDrive:

http://www.bmw.com/com/de/insights/technology/ $/$

technology_guide/articles/idrive.html (accessed 5/2010)
}

or AudiMMI ${ }^{3}$. An introduction to design and evaluation of in-car user interfaces can be found in Burnett et al. [3].

\subsection{Sensors in Vehicles}

Modern cars incorporate a variety of sensors such as accelerometers, distance sensors, cameras, or gyroscopes, which provide access to information inside and outside of cars [9]. Sensor data are mainly used to support and enhance the primary task in the car. Hence, they are used for lane keeping tools [23] and distance systems as well as for different kinds of assistance systems, which try to minimize the risk of collisions and accidents. Prominent examples are parking assistance systems and night vision cameras. In the INTERSAFE project laser scanners are used to track and classify road users and obstacles, providing additional data for the path prediction and risk assessment [7]. Further systems, supporting the driver in an implicit way, include support for acceleration and breaking [24], as well as ABS / ESP. Finally, Pompei et al. [20] presented an integrated system, allowing for assessing and reacting to a driver's cognitive load by tracking his activity.

Additionally, also external (sensor) information is used in cars. The ubiquity of GPS allows for determining the exact position of a car and, in combination with road information, enables route planning. Further approaches exist for obtaining traffic information. These approaches include both offline systems (routes are calculated based on previously collected traffic information and navigation data, e.g., TomTom IQ Routes ${ }^{\mathrm{TM}}$ ) and real-time approaches such as CFCD (Cellular floating phone data system) [22]. CFCD uses timing advance to calculate the distance of a mobile phone to the provider's base station hence triangulating its position. Further sources for real-time traffic information include TomTom HD Traffic ${ }^{\mathrm{TM}}[22]$ and traffic surveillance systems.

\subsection{Car-to-X Communication}

The term Car-to-X (C2X) communication includes both Car-toCar $(\mathrm{C} 2 \mathrm{C})$ and Car-to-Infrastructure (C2I) communication. This type of communication aims at increasing safety in everyday traffic, e.g., by providing real-time information on traffic far beyond what a driver can see [4]. Moreover it enables applications to improve traffic efficiency and infotainment. Whereas $\mathrm{C} 2 \mathrm{C}$ focuses on information exchange between vehicles, C2I is based on communication between vehicles and fixed access points. To realize C2X communication, different architectures have been developed, e.g., VANETS (Vehicular Ad Hoc Networks) [2].

Research in the area of Car-to-X communication aims at closing the gap between onboard devices and long-distance channels (GPS, GSM). In [6] a C2X communication SDK and a software implementation of a networking protocol stack for $\mathrm{C} 2 \mathrm{X}$ and $\mathrm{C} 2 \mathrm{I}$ is introduced. Geocast [5] is an ad hoc routing scheme, which is in Europe considered a core-networking concept for future C2X systems. We refer to [16] for a comprehensive overview on the prerequisites of $\mathrm{C} 2 \mathrm{C}$ communication and interesting technical concepts identified by the $\mathrm{C} 2 \mathrm{C}$ Consortium. With regard to our research $\mathrm{C} 2 \mathrm{X}$ communication is interesting since it allows for realizing IP-based Internet access. This enables access to information on the web, such as news, emails, and YouTube videos.

\footnotetext{
${ }^{3}$ Audi Lexikon MMI

http://www.audi.de/de/brand/de/tools/advice/glossary/ $\$$ mmi.browser.filter_i_m.html (accessed 5/2010)
} 


\section{ONLINE SURVEY}

To assess the potential of in-vehicle micro-entertainment and to gather initial ideas about suitable forms and content we ran an online survey over 2 weeks in February 2010. We recruited people via mailing lists, from friends, colleagues, and Facebook. In total 127 people filled in the survey (92 males, average age 34 years).

\subsection{Results}

First, we were interested in the use of displays in vehicles. More than 90 percent of the people use either a fix or a mobile display for navigation or entertainment purpose.

Second we asked the participants which forms of entertainment would be useful for them (5-Point Likert scale, $1=$ not useful at all, $5=$ very useful, results based on a rating of 4 or 5 , see Figure 1). More than $70.3 \%$ prefer audio, $45.3 \%$ would like to read emails, $40 \%$ could imagine audio augmented with additional images, and $27 \%$ can imagine having short video clips. Short games $(17.2 \%)$ were rather unpopular. The strong preference for audio content (with and without images) might be the result of an adaption to the use of radio in vehicles. Still a significant number of people see an advantage in checking their emails. The rather low popularity of displaying text might be a result of the fact that people prefer having a text read out loudly rather than reading it themselves and that writing on a touch screen inside a car is quite cumbersome. Further suggestions from users regarding content include reading and writing SMS, using vocabulary trainers, displaying RSS feeds, blogs, and playing back longer movies. Surprisingly participants stated that they would even prefer to have the movie played back with audio only (black screen) while driving.

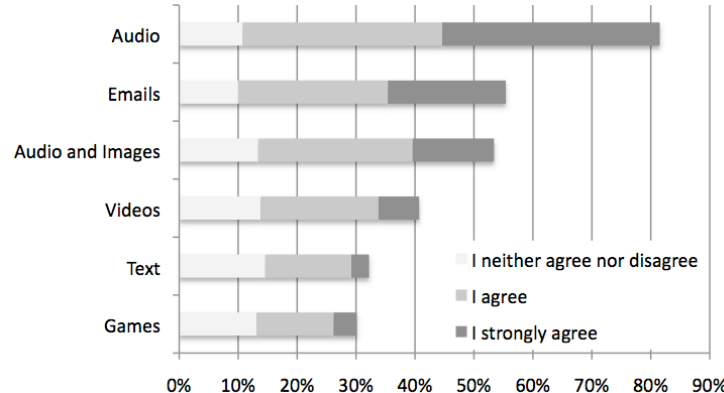

Figure 1: Forms of entertainment suitable for the use in vehicles as rated by the users (5-Point Likert scale, $1=I$ do not agree at all, $5=I$ strongly agree)

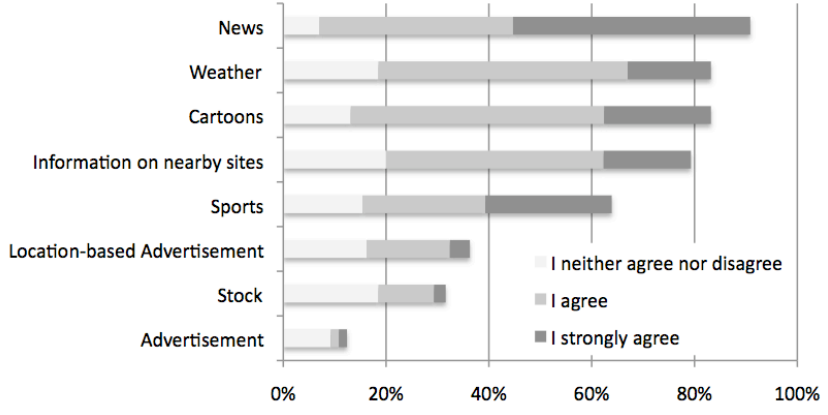

Figure 2: Types of content suitable for in-car entertainment as rated by the users (5-Point Likert scale, $1=I$ do not agree at all, $5=$ I strongly agree)
Third, we were interested in different types of content, participants considered to be attractive (see Figure 2). Most popular were general news $(83.6 \%)$, cartoons $(71.1 \%)$, weather $(64 \%)$, and location-based information on points of interest $(59.4 \%)$. We were also interested in the popularity of showing ads on the screen. Whereas general ads were very unpopular, context-sensitive ads (e.g., related to the car's current location) was considered to be useful by $20.1 \%$. Participants' further suggestions regarding suitable content included appointments, events, regional traffic information, health, and information on close-by restaurants.

Fourth we assessed the acceptance of a system, which adapts content to the length of standing times among the participants of the survey. We found out that more than $50 \%$ would use or definitely use the system (5-Point Likert scale, $1=\mathrm{I}$ would not use the system at all, $5=\mathrm{I}$ would definitely use the system). Based on open-ended questions we found out that most people see the main value of the application in bridging waiting times or using the time in a meaningful way. Further reasons included the provision of compact and up-to-date information as well as entertainment. People who were concerned with using such a system stated that they might feel distracted from traffic and that this might pose a potential security risk. Further issues included that waiting times in front of traffic lights are often too short in order to show meaningful content.

Fifth we asked people in which situations they would consider such a system to be useful. It turned out that $72.4 \%$ would mainly use such a system when being in the car alone, whereas only $16.6 \%$ would use it if other persons were in the car. Further, $58.3 \%$ would prefer the system in case they were familiar with the route, but only $24.4 \%$ in cases they took an unfamiliar route.

\subsection{Discussion}

The results of the online survey reveal a strong potential of microentertainment in vehicles. Whereas audio content is still the preferred form of entertainment, a considerable number of people ( $>$ $40 \%$ ) could already imagine using visual content such as emails, images, or videos in cars. It turned out that content, which could be fit in short time intervals (news, weather, cartoons, etc.), was favored by the participants. When analyzing answers regarding appropriate situations for such a system it becomes clear that commuters would be a primary target group since the majority would use such a system when travelling alone and on familiar routes. Qualitative user feedback revealed that inappropriate length of content would be an issue.

\section{ENABLING MICRO-ENTERTAINMENT}

People spend a considerable amount of their driving time in front of red traffic lights, especially in urban areas. When driving alone, users are seeking for entertainment, e.g., by listening to the radio, calling other people, etc. By providing entertainment in the form of content adapted to standing times we believe that we cannot only enhance the user experience of in-car entertainment in comparison to traditional content (TV program, DVDs), but that we can actively direct the attention of the user back towards the street hence increasing the safety of perceiving content while driving.

In order to realize this knowledge of the waiting time in front of traffic lights is required. While this information could be obtained through communication between the traffic light and the vehicle itself (e.g., Audi's Travolution ${ }^{4}$ ) or from local authorities, current

\footnotetext{
${ }^{4}$ Audi Travolution: http://www.travolution-ingolstadt.de/
} 
approaches lack any standard and are not ubiquitously applicable. Different traffic lights have different red phases and even for the same traffic light often they differ based on the day of the week, or the amount of traffic. Hence we propose a two-step approach to provide entertaining content in a meaningful way. First, we show how to detect, refine, and extend areas in front of traffic lights. Second, we create an estimation of the waiting time for each traffic light enhanced with information of each car approaching.

\subsection{Identifying Traffic Light Zones}

In a first step we identify areas in front of traffic lights, so-called Traffic Light Zones (TLZs). We use GPS information, which allows for applying the approach independent of a traffic light's location, the day, and the time of day. We assess how long red phases are based on the standing times of the vehicle. Therefore we do not even need to know the location of a traffic light beforehand but rather learn it depending on the stop-and-go characteristics of a GPS track. Based on the GPS data we obtain a vehicle's velocity in order to determine whether it is moving or not. Since the accuracy of GPS data is not sufficient for a precise calculation of position and velocity we use state transitions and a threshold to determine a vehicle's state. We distinguish the following states: (1) Vehicle is moving and (2) Vehicle is standing (Figure 3).

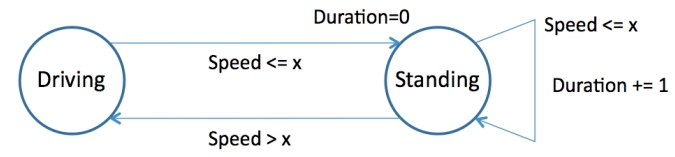

Figure 3: State transitions of a vehicle's status. (1) Vehicle is moving, (2) vehicle is standing

Standing and moving times are defined as the time between two state transitions. Additionally we store the direction for each standing point as a tuple, consisting of longitude and latitude (dir_long, dir_lat). To calculate the correct direction of travel we consider a reference point, stored at a certain frequency. To create a TLZ we allocate standing points in front of the same traffic light. We do so by calculating the distance between a vehicle's current standing point and existing TLZs. For calculating the TLZ we use the following formulas based on Pythagoras' theorem:

$$
\begin{aligned}
& \text { lat }=\frac{\text { lat }_{1}+\text { lat }_{2}}{2} \\
& d x=111.13 \mathrm{~km} \cdot \cos (\text { lat }) \cdot\left(\text { lon }_{1}-\text { lon }_{2}\right) \\
& d y=111.13 \mathrm{~km} \cdot\left(\text { lat }_{1}-\text { lat }_{2}\right) \\
& \text { distance }=\sqrt{\mathrm{dx}^{2}+d y^{2}}
\end{aligned}
$$

\section{Equation 1: Calculation of distance between standing point and $T L Z^{5}$}

To calculate the direction we compare longitude and latitude of the reference point with the standing point. In case of a match with the previously stored zone, we assume an equal direction.

\subsubsection{Algorithm}

The algorithm used for identifying traffic light zones is depicted in Figure 4. Once the state changes to "vehicle is standing" we test if a zone exists, which lies within a certain distance from the

\footnotetext{
${ }^{5}$ Note: Whereas for the latitude the distance between two degrees is always $111.13 \mathrm{~km}$, it varies for the longitude, ranging from 0 $\mathrm{km}$ at the poles to $111.13 \mathrm{~km}$ at the equator.
}

vehicle's position. If yes, we also test the direction and in case of a match associate the current standing point with the TLZ, and expand it. Hence, we are able to create large TLZs, e.g., in front of traffic lights producing long congestions. In case no traffic light position lies within the pre-defined distance or if the direction does not match, a new zone is created. We use 3 variables which impact on how precisely TLZs are created and rediscovered.

Velocity threshold: The threshold specifies below which value the velocity of a vehicle has to drop for considering it as a state transition. Values between $0-1 \mathrm{~km} / \mathrm{h}$ hardly occur in GPS devices since the velocity asymptotically adapts to the correct speed. Hence, the velocity after 5 seconds still lay beyond $0.5 \mathrm{~km} / \mathrm{h}$.

TLZ size: This parameter specifies within which distance to a given zone a new standing point has to lie in order to be considered a part of it. Low values lead to more TLZs, high values might incorrectly identify standing points as a part of a traffic light zone.

Update frequency of directional reference point: for calculating the direction of travel, a reference point is used. This reference point is a previously stored position of the car. The update frequency specifies how often the reference point is stored.

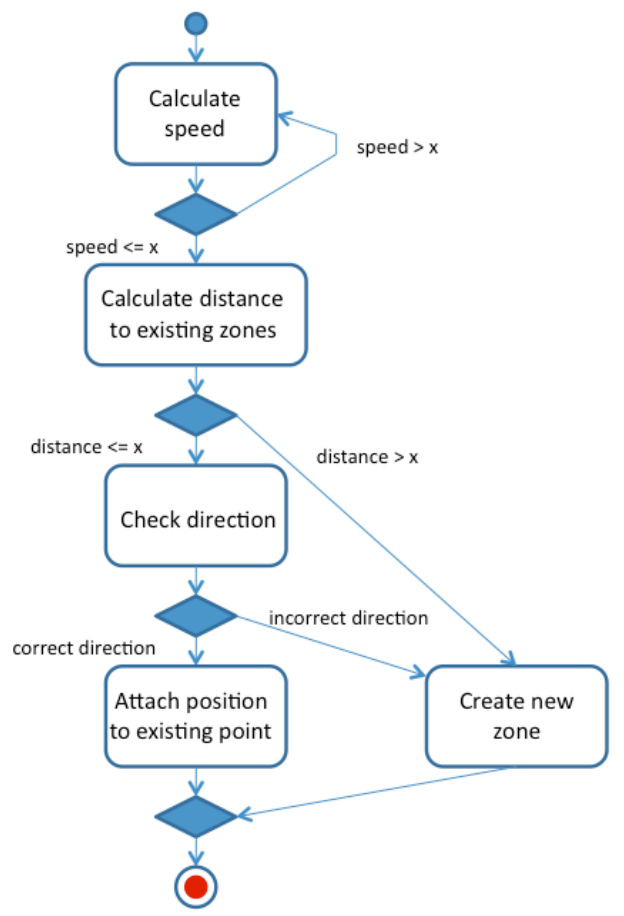

Figure 4: Algorithm for identifying traffic light zones (TLZ)

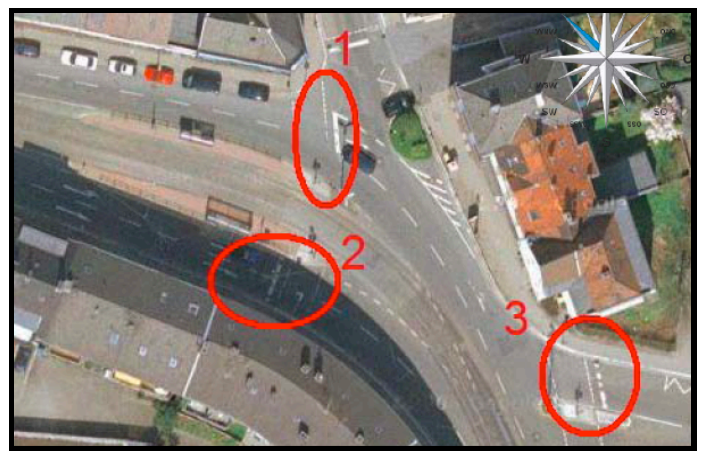

Figure 5: Correct position of the traffic lights 
To understand the impact of those variables and to obtain suitable values we equipped two daily commuters with GPS loggers and had them log their GPS track over the course of 2 weeks. We collected a total of 36 GPS tracks ( 9 GPS tracks for each participant commuting from home to work and vice versa). We applied our algorithm to the data and tested different values for the size of the zone and the frequency of storing the reference point in order to obtain close-to-optimal results. For a better evaluation we visualized the results using the Google Maps API. Figure 5 shows the correct location of the traffic lights, the results are depicted in Figure 6. Markers represent positions where the first standpoint of a TLZ was defined; rectangles represent the enclosing area of the maximum and minimum longitude and latitude values.

Configuration 1 gives an example for a too large TLZ size. The TLZ size parameter leads to an overlap of traffic lights 1 and 2 . Whereas the extension towards the southwest is a result of imprecise GPS data (street canyons) the extension towards the east is a result of the left-turning traffic. Configuration 2 resulted in 5 TLZs. Zones B, F, and A are associated with traffic lights 1, 2, and 3. Zone $\mathrm{G}$ represents the left-turning lane of traffic light 2. Zone $\mathrm{L}$ is in the same location as the top-corner of $\mathrm{C}$ in configuration 1 . It is correctly not associated with zone $\mathrm{B}$ since this point is approached by northbound traffic. The outliers in zones A (north) and B (south) are again a result of imprecise GPS data. Configuration 3 resulted in 2 zones. Due to the higher update frequency of the reference point the zones around traffic lights $\mathrm{A}$ and $\mathrm{B}$ are merged, as are left-turning traffic from traffic light 2 and 3 . The reference point is too close to the current position.

\subsubsection{Results}

Figure 6 shows that the size of a zone and the update frequency of the reference point for the direction have a strong impact on the correct association of the points with existing TLZs. In total configuration 2 (zone size $=30 \mathrm{~m}$, update frequency $=6 \mathrm{~Hz}$ ) returned the best results. Further tests revealed that a lower update frequency led to wrong results as the probability for changes in the direction increased. We discovered that values below $0.2 \mathrm{~km} / \mathrm{h}$ generated less standing points and entire zones collapsed. Values between $0.2-1 \mathrm{~km} / \mathrm{h}$ resulted in a lower standing time in the zones. An increase in the velocity also led to an increase in the standing times. A threshold of $1 \mathrm{~km} / \mathrm{h}$ turned out to be a good compromise.

\subsubsection{Discussion}

Though we envision our approach to work in most cases we are not able to distinguish between vehicles stopping in front of traffic lights, turning areas, parking lots, traffic jams, stop-and-go traffic, and at railway crossings. Whereas traffic-jams, parking, and stopand-go traffic only pose a minor issue, all types of stops which are likely to occur frequently are a problem since they might be identified as a traffic light zone by the algorithm.

\subsection{Estimation of Waiting Times}

On average, entire red phases last 30-45 seconds. However, precise knowledge about the length can be used in various ways. First, content can be tailored to fit the waiting time. Second, drivers could be actively made aware of resuming traffic.

For the calculation of the standing time in front of traffic lights we first calculate the mean value $X$ of all previously stored standing times. Second, we calculate the standard deviation $s$ on the variance of all known data. Thus we get as a result an interval $[X-s, X+s]$, which represents the expected duration of the next standing time. In order to avoid that the duration of the clips ex- tends the duration of the standing time we opted to use the interval $[X-s, X]$ in the prototype. The maximum length of the clip was set to $X-(s / 2)$ making sure that the clip length neither exceeded nor to a large extent fell below the actual waiting time.

The approach's quality depends on the size of the dataset. Whereas small samples are likely to produce high error rates, large samples provide a good approximation of the standing time. The value of the standard deviation can be seen as a measure for quality - the higher the amount, the worse is the data quality. To more quickly obtain larger sets of data we store information in a central database hence making it available to all other drivers.

Though our algorithm returns correct results in front of standard traffic lights the algorithm is not yet able to adjust the estimated waiting time to adaptive traffic lights (traffic lights, using metal detectors and motion sensors in order to detect approaching cars). Further, we do not yet consider different times of the day.

\section{IMPLEMENTATION}

Our prototype system is based on a client-server infrastructure. We implemented 2 clients for the use on laptops and on mobile phones. Both clients are written in Python. The following chapter provides an overview of requirements and the system architecture.

\subsection{Requirements}

The following requirements have to be satisfied:

Determine vehicle status: The system logic needs to be able to distinguish whether a car is moving or not. Due to shortcomings with the GPS-based velocity information, state transitions are used to determine the vehicle's status.

TLZ management: Standing points need to be associated with TLZs by retrieving existing ones from a central database or new TLZs have to be created and inserted. For existing TLZs the system needs to be capable of recognizing that a vehicle stopped in the same TLZ several times and adapt the estimated waiting time. After each stop, the waiting time needs to be updated in the DB.

Content management: Based on the estimated waiting time content of appropriate length has to be selected. Content is to be stored either locally or retrieved from the Internet. In order to increase the user experience, users should be able to specify their interests or explicitly select types of content to be shown.

Offline Mode: The system needs to be functional without Internet connection as in tunnels or in rural areas coverage may be bad. A local DB is used to synchronize regularly with a central DB.

Feedback: The system should inform other users standing in the same traffic light zone about a change of status (e.g., that the traffic light is expected to change back to green again). Additionally, the user must be able to abort playback and information on these actions need to be stored and handled accordingly.

\subsection{Hardware}

For our prototype we used the Bluemax Bluetooth GPS-4043 recorder as a GPS receiver/logger. The accuracy is specified with $3 \mathrm{~m}$, the maximum frequency is below $1 \mathrm{~s}$. For the mobile client we used a Nokia 5800 XpressMusic with a pre-installed Python for S60 runtime environment as well as a Python Script Shell 1.4.5.

\subsection{System Architecture}

On our server we use MySQL to store TLZs and timing information. The client-server communication is based on PHP. Local (client-side) database information is stored in XML. TLZs are rep- 
resented by longitude and latitude, sum of stops, average standing time, longitude and latitude of the direction, maximum and minimum standing time, and standard deviation. We use an application server to realize access between clients and database. Both clients provide similar functionality. They read the current location and velocity from a GPS receiver connected via Bluetooth. In case the velocity drops below the threshold, required data is accessed from the local or remote DB. The clients support two modes:

Online Mode: Once the vehicle stops the current location and direction is transferred to the database. Based on the retrieved estimated waiting time suitable content is selected for durations of more than 10 seconds. The application server provides a getStatus() method for each traffic light. It returns its current status and is used to stop the playback. The same is true if the vehicle starts moving. Finally, the waiting time is written to the DB.

Offline Mode: Upon the state transition to "vehicle is standing" the local XML database is checked for the existence of a TLZ at the given location and direction and the decision is made whether content is played back or not. When the zone is left, either the average duration spent in the current zone is recorded or a new zone is being initiated. The currently played back content is stopped.

\subsection{Content}

Once our application recognizes that a vehicle came to a halt, we check in the database if a TLZ exists in the current location. In case the expected standing time exceeds 10 seconds (we consider this to be the minimum time to display meaningful content), we select content of appropriate length from a local set. A more sophisticated version could preload content based on the taken route (this information could be extracted from a navigation system) and pre-load the content, hence making it possible to download up-to-date content on the fly while driving.

\section{QUALITATIVE EVALUATION}

To evaluate the system we conducted several test drives. As content we used audio clips in combination with still images to ensure a minimal distraction of the driver. The length of the clips varied between 8 and 32 seconds (fitting $X-(s / 2)$, where $X \geq 10$ s). Depending on the estimated standing time a clip was chosen and played back. Clips were previously stored on the mobile device.

\subsection{Testing Previously Tagged Routes}

For the first evaluation we had our test person drive a route where we previously created TLZs based on a GPS log. For the drive we logged the standing times in front of red traffic lights and whether the clip was played back, and compared the duration with the actual standing time. Figure 7 depicts a part of the evaluation route. All markers represent positions with state transitions to "vehicle is standing". In Table 1 a comparison between the estimated standing time (values from database), the actual standing time (obtained by using a stop watch), and the length of the played clip is given. In order to test which TLZ a standing point was assigned to the TLZs were checked and associated based on timestamps.

Results: For each standing point a TLZ was created, the standing point associated with the TLZ and a media clip played back if applicable. Whereas zones $1-12$ are actually traffic lights, zone 13 is a left-turn lane. The clip was played back twice due to the stopand-go of the vehicle. For the zones 4, 9, 10, and 13 the media clip was longer as the actual standing time. The standing time in zones 4, 9, and 13 was below 4 seconds. To solve this issue a delay could be added at the beginning of the clip, hence making the
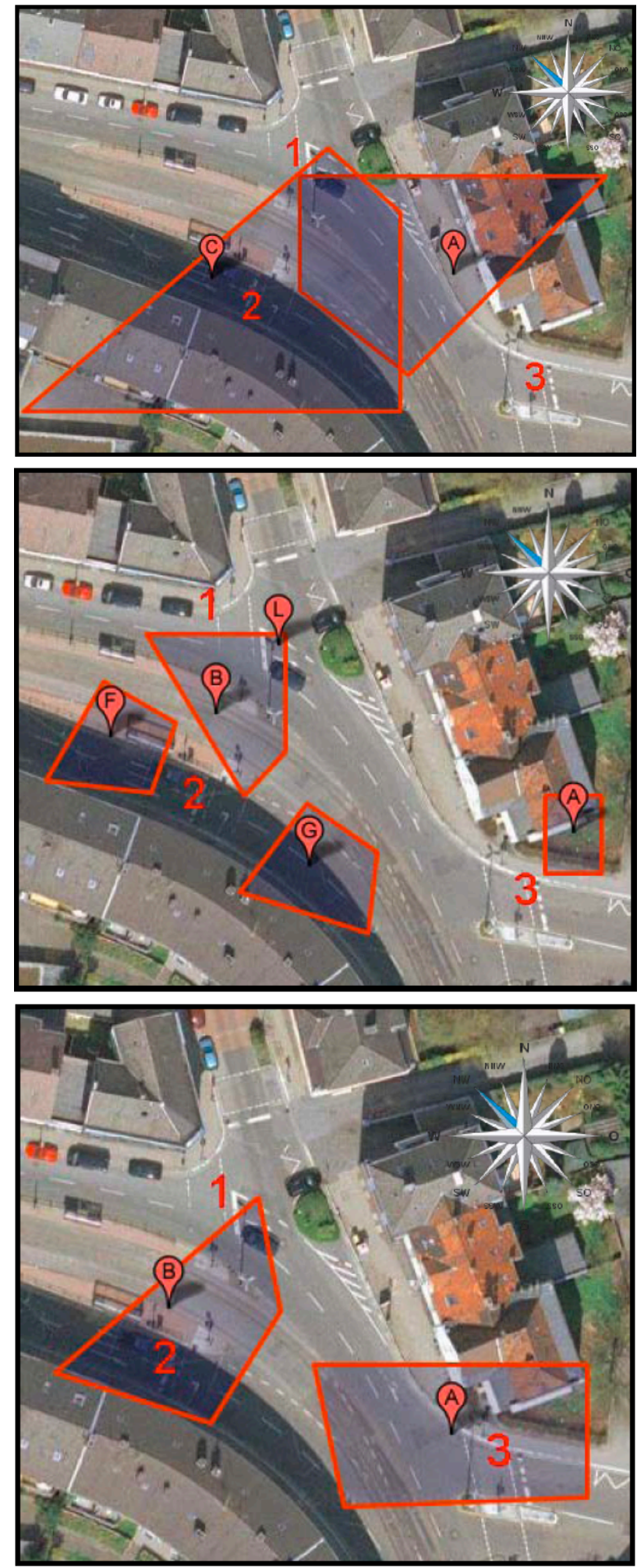

Figure 6: (a) Configuration 1: zone size $=50 \mathrm{~m}$, update frequency for reference point $=6 \mathrm{~Hz}$

(b) Configuration 2: zone size $=30 \mathrm{~m}$, update frequency $=6 \mathrm{~Hz}$ (c) Configuration 3: zone size $=30 \mathrm{~m}$, update frequency $=12 \mathrm{~Hz}$ 


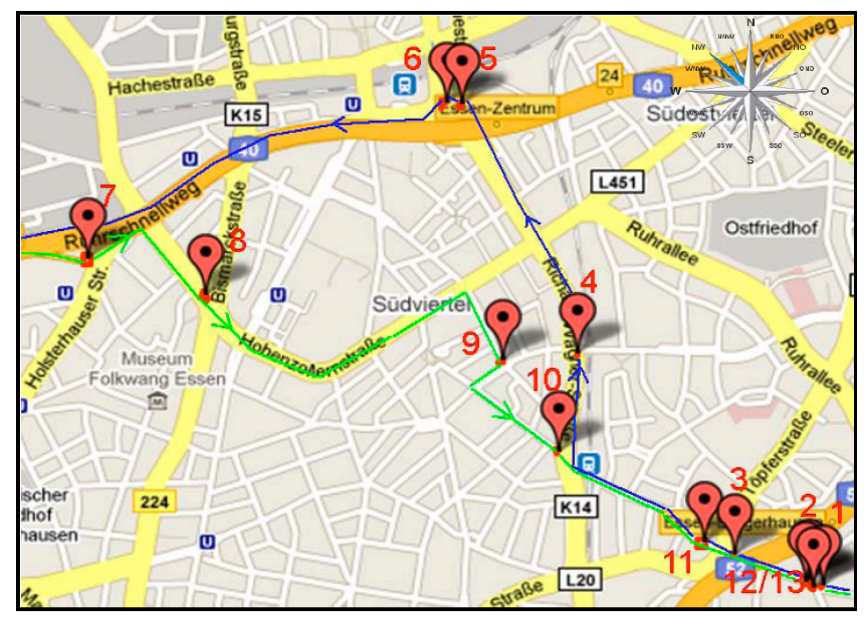

Figure 7: Route with previously created TLZs. The red markers represent state transitions (vehicle is standing).

\begin{tabular}{|c|c|c|c|}
\hline $\begin{array}{c}\text { TLZ } \\
\mathbf{N r}\end{array}$ & $\begin{array}{c}\text { Estimated } \\
\text { standing time }\end{array}$ & $\begin{array}{c}\text { Actual stand- } \\
\text { ing time }\end{array}$ & $\begin{array}{c}\text { Length of selected } \\
\text { media clip }\end{array}$ \\
\hline $\mathbf{1}$ & $26,8 \mathrm{~s}$ & $32 \mathrm{~s}$ & $27 \mathrm{~s}$ \\
\hline $\mathbf{2}$ & $10,9 \mathrm{~s}$ & $15 \mathrm{~s}$ & $12 \mathrm{~s}$ \\
\hline $\mathbf{3}$ & $17,8 \mathrm{~s}$ & $28 \mathrm{~s}$ & $17 \mathrm{~s}$ \\
\hline $\mathbf{4}$ & $8,6 \mathrm{~s}$ & $3 \mathrm{~s}$ & $8 \mathrm{~s}$ \\
\hline $\mathbf{5}$ & $9,1 \mathrm{~s}$ & $12 \mathrm{~s}$ & $8 \mathrm{~s}$ \\
\hline $\mathbf{6}$ & $14,1 \mathrm{~s}$ & $20 \mathrm{~s}$ & $12 \mathrm{~s}$ \\
\hline $\mathbf{7}$ & $25 \mathrm{~s}$ & $34 \mathrm{~s}$ & $27 \mathrm{~s}$ \\
\hline $\mathbf{8}$ & $20,7 \mathrm{~s}$ & $18 \mathrm{~s}$ & $23 \mathrm{~s}$ \\
\hline $\mathbf{9}$ & $9,0 \mathrm{~s}$ & $2 \mathrm{~s}$ & $8 \mathrm{~s}$ \\
\hline $\mathbf{1 0}$ & $32,7 \mathrm{~s}$ & $24 \mathrm{~s}$ & $32 \mathrm{~s}$ \\
\hline $\mathbf{1 1}$ & $39,2 \mathrm{~s}$ & $48 \mathrm{~s}$ & $32 \mathrm{~s}$ \\
\hline $\mathbf{1 2}$ & $27,5 \mathrm{~s}$ & $30 \mathrm{~s}$ & $27 \mathrm{~s}$ \\
\hline $\mathbf{1 3}$ & $9,9 \mathrm{~s}$ & $3 \mathrm{~s}$ & $8 \mathrm{~s}$ \\
\hline $\mathbf{1 3}$ & $9,9 \mathrm{~s}$ & $2 \mathrm{~s}$ & $8 \mathrm{~s}$ \\
\hline
\end{tabular}

Table 1: Comparison of estimated and actual standing time.

playback shorter but more reliable. In zone 3 and 11 the clip was more than 10 seconds shorter than the actual standing time. For zone 11, the standing time exceeded the maximum clip length, for zone 3 the average standing time was below the actual standing time. In total a majority of the TLZs were detected correctly and playback times matched the standing times quite well.

\subsection{Testing Previously Untagged Routes}

We had the same person make an evaluation drive on a route with no previously stored information on TLZs (Figure 8). The route was driven in total four times. For the first 3 drives we used the offline mode with an empty XML database, for the last drive we synchronized the XML database with our central database. The fourth drive was conducted in online mode in order to test whether TLZs are built correctly and if synchronization works. During the drive we logged information on position of standing points, the standing duration and potentially played back clips. Markers are again positions where the vehicle stopped. Blue and green colors indicate the driving direction. Table 2 gives an overview of the actual and the average standing time. All bold entries resulted in the creation of a new TLZ. Out of the TLZs, 8 were correctly created in front of traffic lights, zone 7 was created due to a traffic jam and 10 and 11 while waiting behind a tram.

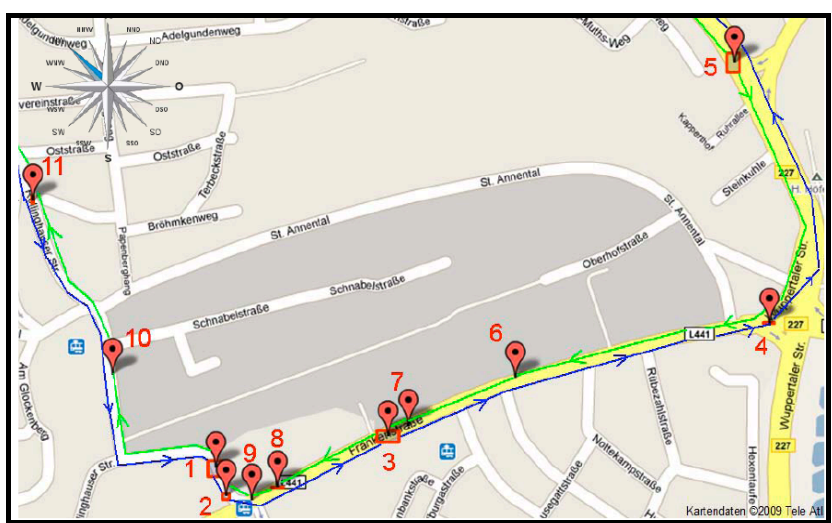

Figure 8: Evaluation drive on an untagged route. The red markers represent state transitions (vehicle is standing).

\begin{tabular}{|c|c|c|c|c|c|}
\hline $\begin{array}{c}\text { TLZ } \\
\text { Nr }\end{array}$ & Run 1 & Run 2 & Run 3 & Run 4 & $\begin{array}{c}\text { Average standing } \\
\text { time }\end{array}$ \\
\hline $\mathbf{1}$ & - & - & - & $\mathbf{5 s} / 33 \mathrm{~s}$ & $19 \mathrm{~s}$ \\
\hline $\mathbf{2}$ & $\mathbf{2 8 s}$ & $14 \mathrm{~s}$ & $19 \mathrm{~s}$ & $21 \mathrm{~s}$ & $20,5 \mathrm{~s}$ \\
\hline $\mathbf{3}$ & $\mathbf{2 0 s}$ & $6 \mathrm{~s}$ & $11 \mathrm{~s}$ & - & $12,333 \mathrm{~s}$ \\
\hline $\mathbf{4}$ & $\mathbf{5 9} \mathrm{s}$ & $47 \mathrm{~s}$ & $57 \mathrm{~s}$ & $49 \mathrm{~s}$ & $53 \mathrm{~s}$ \\
\hline $\mathbf{5}$ & $\mathbf{1 4 s}$ & $10 \mathrm{~s}$ & $14 \mathrm{~s}$ & $18 \mathrm{~s}$ & $14 \mathrm{~s}$ \\
\hline $\mathbf{6}$ & - & $\mathbf{1 2 s}$ & - & - & $12 \mathrm{~s}$ \\
\hline $\mathbf{7}$ & - & - & $\mathbf{1 1 s}$ & - & $11 \mathrm{~s}$ \\
\hline $\mathbf{8}$ & - & $\mathbf{1 1 s}$ & $11 \mathrm{~s}$ & - & $11 \mathrm{~s}$ \\
\hline $\mathbf{9}$ & - & - & - & $\mathbf{9 s}$ & $9 \mathrm{~s}$ \\
\hline $\mathbf{1 0}$ & - & - & $\mathbf{1 8 s}$ & - & $18 \mathrm{~s}$ \\
\hline $\mathbf{1 1}$ & - & $\mathbf{1 1 s}$ & $14 \mathrm{~s}$ & - & $12,5 \mathrm{~s}$ \\
\hline
\end{tabular}

Table 2: Comparison between actual and estimated standing time for all 4 runs in 10 TLZs

Results: The creation of TLZs worked correctly in most of the cases, the synchronization as well as online and offline mode worked smoothly. As expected the initial building of zones at standing points caused problems since TLZs were also created in locations without traffic lights.

\subsection{User Feedback}

Qualitative user feedback revealed that there might in fact be an effect on users' driving behavior. Our test driver (male, 28 years) stated that he deliberately reduced his use of the engine break and that avoided approaching a traffic light slowly. Instead he tried to halt only in front of the traffic light. We believe that this is because the test driver was keen on seeing the next media clip. Whereas these findings are certainly not representative they give an idea how users' behavior might be affected.

\section{FUTURE WORK}

The current implementation has several shortcomings, which we plan to address in future versions. First, there is no way yet to verify if the system estimates the waiting time correctly. In an online version information from the car in front could be used to make the systems aware that the traffic light turned green again and automatically end the content. Another option could be to integrate a camera for detecting traffic lights.

Second, in the current implementation the clip can be aborted by using a stop button but this information is not being assessed. However, this might serve as an indicator that the current zone is a traffic light but rather at a yield sign or railroad crossing. 
Third, a system could learn the length of red phases without information from the database. Once a car is stopping for the second time it can be assumed that the duration of the next standing interval is as long as the previous one if it occurs within the same TLZ.

Finally we envision a further enhancement of our system's accuracy through the advent of new technologies, such as Galileo. Also obtaining CAN bus information directly from the vehicle (e.g., current velocity) could increase the data quality.

\section{CONCLUSION}

In this paper we presented a context-based approach, which enables the learning of standing points and the estimation of waiting times in order to tailor content on in-car displays. We consider this work to be the first step towards safer car entertainment. By tailoring the length of content to the estimated waiting time in cars we do not only enable a more pleasant driver experience but also allow for making drivers aware when attention should be directed back towards the road. We discussed several challenges, e.g., the distinction between different phenomena in daily traffic that cause vehicles to stop, such as traffic jams, railroad crossings, or parking lots. Yet, our algorithm produces decent results.

Evaluating in-car entertainment in the real world is a difficult challenge, since safety might be compromised in case of a malfunction of the system or of the GPS device (street canyons, etc.). We conducted a small-scale, qualitative evaluation with nondistractive content (audio and still images), which indicated that there might indeed be a quite significant influence on how drivers approach traffic lights. However, we are currently working on getting a large-scale user study on the way. We are especially interested in the degree of distraction (we plan to do an eye-tracking study in a car simulator), but also plan a longer-term field study were people use an entertainment system over the course of several weeks. Initial findings indicate, that especially for daily commuters, such a system might be of value and that it has the potential to increase road safety.

\section{REFERENCES}

1. Alt, F., Sahami Shirazi, A., Pfeiffer, M., Holleis, P., Schmidt, A. TaxiMedia: An Interactive Context-Aware Entertainment and Advertising System. In Proc. of 2nd Pervasive Advertising Workshop (2009). Lübeck, Germany.

2. Boukerche, A., Oliveira, H., Nakamura, E. and Loureiro, A. Vehicular ad hoc networks: a new challenge for localizationbased systems. In: Computer Communications (2008) Vol. 31.

3. Burnett, G. Designing and evaluating in-car user interfaces. In: Lumsden, J. Handbook of research on user-interface design and evaluation for mobile technology (2008). Idea Group Inc.

4. Dashtinezhad, S., Nadeem, T., Dorohonceanu, B., Borcea, C., Kang, P. and Iftode, L. TrafficView: a driver assistant device for traffic monitoring based on car-to-car communication. In Proc. of Vehicular Technology Conference, VTC'04 (2004).

5. Festag, A., Baldessari, R., Zhang, W., Le, L., and Sarma, A.. CAR-2-X Communication for Safety and Infotainment in Europe. In: NEC Technical Journal (2008). Vol. 3,1

6. Festag, A., Baldessari, R., Zhang, W., and Le, L. Car-2-X Communication SDK - A Software Toolkit for Rapid Application Development and Experimentations. In Proc. of IEEE VehiMobil (2009). Dresden, Germany.
7. Fuerstenberg, K.C. and Roessler, B. Advanced Intersection Safety - The EC project INTERSAFE. In Proc. of IV'06 (2006), San Diego, USA.

8. Funkhouser, D. and Chrysler, S. Assessing Driver Distraction Due to In-Vehicle Video Systems through Field Testing at the Pecos Research and Testing Center. (2007)

9. Gharavi, H., Venkatesh Prasad, K. and Ioannou, P. Scanning Advanced Automobile Technology. In Proc. of IEEE, 95(2) (2007). pp. 328-333.

10. Green, P. Where do drivers look while driving (and for how long). In: Human factors in traffic safety (2002). pp. 77-110.

11. Gustafsson, A., Bichard, J., Brunnberg, L., Juhlin, O. and Combetto, M. Believable environments: generating interactive storytelling in vast location-based pervasive games. In Proc. of ACE '06 (2006), vol. 266. ACM, New York.

12. Harbluk, J.L., Noy, Y.I., and Eizenman, M. The impact of cognitive distraction on driver visual behaviour and vehicle control. Transport Canada (2002).

13. Hills, B. Vision, visibility, and perception in driving. In: Perception (1980). 9(2), p. 183.

14. Juhlin, O. The Interactive Road. In: Innovation Magazine (2008), Vol. 8(3). pp. 36-37.

15. Kamp J.F., Marin-Lamellet C., Forzy J.F. and Causeur D. HMI aspects of the usability of Internet services with an in-car terminal on a driving simulator. In: Journal of International Association of Traffic and Safety Sciences (2001). Vol. 25,2.

16. Kosch, T. Technical Concept and prerequisites of car-to-car communication. Technical report, BMW Group R\&T (2002).

17. Langer, G. A Look Under the Hood of a Nation on Wheels. In: ABC News/Time magazine/Washington Post poll (2005). http://abcnews.go.com/images/Politics/973a2Traffic.pdf

18. Laurier, E., Lorimer, H., Brown, B., Jones, O., Juhlin, O., Noble, A., Perry, M., Pica, D., Sormani, P., Strebel, I., Swan, L., Taylor, A. S. Driving and passengering: notes on the ordinary organization of car travel. Mobilities, (2008) 3(1), pp. 1-23.

19. Palazzi, C.E., Roccetti, M., Ferretti, S., Pau, G., and Gerla, M. Online games on wheels: Fast game event delivery in vehicular ad-hoc networks. In Proc. of Vehicle-to-Vehicle Communications (2007). Istanbul, Turkey.

20. Pompei, F., Sharon, T., Buckley, S. and Kemp, J. An automobile-integrated system for assessing and reacting to driver cognitive load. In Proc. of Convergence'02 (2002). pp. 21-23.

21. Tester, J., Fogg, B., and Maile, M. 2000. CommuterNews: a prototype of persuasive in-car entertainment. In CHI '00 Extended Abstracts (2000). The Hague. pp. 24-25.

22. TomTom. White paper - How TomTom's HD Traffic ${ }^{\mathrm{TM}}$ and IQ Routes ${ }^{\mathrm{TM}}$ data provides the very best routing"; http://www.tomtom.com/lib/doc/download/ $\psi^{\mathbb{}}$ HDT_White_Paper.pdf (accessed 5/2010)

23. Wu, S.J., Chiang, H.H., Perng, J.W., Lee, T.T. and Chen, C.J. The automated lane-keeping design for an intelligent vehicle. In Proc. of IEEE Intelligent Vehicles (2005). pp. 508-513.

24. Zhaohui, W., Yanfei L. and Gang, P. A Smart Car Control Model for Brake Comfort Based on Car Following. In: Intelligent Transportation Systems, IEEE Transactions (2009), 10(1), pp. 42-46. 